\title{
Drawing the Line: Differentiating Transudates from Exudates
}

\author{
Veena B. Antony \\ R.L. Roudebush VA Medical Center, Pulmonary and Critical Care Medicine, Indiana University School of Medicine, \\ Indianapolis, Ind., USA
}

Involvement of the pleural mesothelium in inflammatory disease processes results in the development of an exudative pleural effusion. When the pleura itself is not inflamed and there is increased intravascular pressure, transudative effusions occur. This important concept is clearly illustrated in the paper by Alexandrakis and Bouros [1] in this issue of Respiration. They evaluated the level of interleukin- $1 \beta$ in pleural fluids of a large number of patients and found it lacking in both sensitivity and specificity when compared to other criteria for the differentiation of transudates from exudates. In a refreshingly candid discussion, the authors point out that although cytokines can be adequate markers for pleural effusion differentiation, they are not as sensitive, specific, or cost and time efficient as the standard tests that are easily available.

Drawing the line between transudates and exudates an be an exercise in reductionism and simplistic thinking. A transudative effusion can hide a serious disease such as atelectasis secondary to a pulmonary embolism, while an exudate in a patient with heart failure may be secondary to treatment with diuretics. It is therefore critical to recognize that differentiation between transudates and exu- dates is artificial and not diagnostic for a particular disease entity. Rather, attention should be paid to the patient's clinical presentation to help narrow the differential diagnosis of the pleural effusion.

It is also time to draw the line on the study of cytokines as a method to differentiate between transudates and exudates. Once the pleural mesothelium has initiated an inflammatory cascade, multiple cytokines, either from the mesothelium, underlying tissues, or the recruited inflammatory cells, cause an increasing ripple-like effect of cytokines in the pleural space. As the authors of the paper clearly point out, the presence of cytokines in the pleural fluid indicate pleural pathology but should not be used as a routine laboratory test for discriminating between transudates and exudates.

Reference $\quad 1$ Alexandrakis M, Kyriakou D, Alexandraki R,

Pappa KA, Antonakis N, Bouros D: Pleural
interleukin-1- $\beta$ in differentiating transudates
and exudates: Comparative analysis with other
biochemical parameters. Respiration 2002;69:
201-206.

Veena B. Antony, MD

Professor of Medicine and Pediatrics, Indiana University School of Medicine Chief, Pulmonary and Critical Care Medicine, R.L. Roudebush VA Medical Center 1481 West 10th Street, Indianapolis, IN 46202 (USA)

Tel. +1 317554 0036, Fax +1 317554 0262, E-Mail vantony@iupui.edu 